\title{
APPROXIMATION OF STATIONARY STATISTICAL PROPERTIES OF DISSIPATIVE DYNAMICAL SYSTEMS: TIME DISCRETIZATION
}

\author{
XIAOMING WANG
}

\begin{abstract}
We consider temporal approximation of stationary statistical properties of dissipative infinite-dimensional dynamical systems. We demonstrate that stationary statistical properties of the time discrete approximations, i.e., numerical scheme, converge to those of the underlying continuous dissipative infinite-dimensional dynamical system under three very natural assumptions as the time step approaches zero. The three conditions that are sufficient for the convergence of the stationary statistical properties are: (1) uniform dissipativity of the scheme in the sense that the union of the global attractors for the numerical approximations is pre-compact in the phase space; (2) convergence of the solutions of the numerical scheme to the solution of the continuous system on the unit time interval $[0,1]$ uniformly with respect to initial data from the union of the global attractors; (3) uniform continuity of the solutions to the continuous dynamical system on the unit time interval $[0,1]$ uniformly for initial data from the union of the global attractors. The convergence of the global attractors is established under weaker assumptions. An application to the infinite Prandtl number model for convection is discussed.
\end{abstract}

\section{INTRODUCTION}

Many dissipative dynamical systems arising in physical applications possess very complex behavior with abundant instability and sensitive dependence on initial data and parameters $[32$. It is well known that statistical properties of these kinds of systems are much more important, physically relevant and stable than single trajectories [12, 19, 21, 23, 24, 36.

For a given abstract autonomous continuous-in-time dynamical system determined by a semi-group $\{S(t), t \geq 0\}$ on a separable Banach space $H$, we recall that if the system reaches a statistical equilibrium in the sense that the statistics are time-independent (stationary statistical properties), the probability measure $\mu$ on $H$ that describes the stationary statistical properties can be characterized via either the strong (pull-back) or weak (push-forward) formulation [12, 21, 23, 36, 37.

Definition 1 (Invariant Measure (Stationary Statistical Solution)). Let $\{S(t), t \geq$ $0\}$ be a continuous semi-group on a Banach space $H$ which generates a dynamical

Received by the editor September 12, 2008 and, in revised form, December 23, 2008.

2000 Mathematics Subject Classification. Primary 65P99, 37M25, 65M12, 37L40, 76F35, 76F20, 37L30, 37N10, 35Q35.

Key words and phrases. Stationary statistical property, invariant measure, global attractor, dissipative system, time discretization, uniformly dissipative scheme, infinite Prandtl number model for convection, Nusselt number.

(C)2009 American Mathematical Society Reverts to public domain 28 years from publication 
system on $H$. A Borel probability measure $\mu$ on $H$ is called an Invariant Measure (Stationary Statistical Solution) of the dynamical system if

$$
\mu(E)=\mu\left(S^{-1}(t)(E)\right), \forall t \geq 0, \forall E \in \mathcal{B}(H),
$$

where $\mathcal{B}(H)$ represents the $\sigma$-algebra of all Borel sets on $H$.

Equivalently, the invariant measure $\mu$ can be characterized through the following push-forward weak invariance formulation:

$$
\int_{H} \Phi(\mathbf{u}) d \mu(\mathbf{u})=\int_{H} \Phi(S(t) \mathbf{u}) d \mu(\mathbf{u}), \forall t \geq 0
$$

for all bounded continuous test functionals $\Phi$.

An invariant measure (stationary statistical solution) for a discrete dynamical system generated by a map $S_{\text {discrete }}$ on a Banach space $H$ is defined in a similar fashion with the continuous time $t$ replaced by discrete time $n=0,1,2, \ldots$.

Another popular object utilized below and associated with the long time behavior of a dynamical system is the global attractor, which we recall for convenience 12, 14, 32.

Definition 2 (Global Attractor and Dissipative System). Let $\{S(t), t \geq 0\}$ be a continuous semi-group on a Banach space $H$ which generates a continuous dynamical system on $H$. A set $\mathcal{A} \subset H$ is called the global attractor of the dynamical system if the following three conditions are satisfied.

(1) $\mathcal{A}$ is compact in $H$.

(2) $\mathcal{A}$ is invariant under the flow, i.e.

$$
S(t) \mathcal{A}=\mathcal{A} \text {, for all } t \geq 0 .
$$

(3) $\mathcal{A}$ attracts all bounded sets in $H$, i.e., for every bounded set $B$ in $H$,

$$
\lim _{t \rightarrow \infty} \operatorname{dist}_{H}(S(t) B, \mathcal{A})=0 .
$$

Here, dist $_{H}$ denotes the Hausdorff semi-distance in $H$ between two subsets, which is defined as

$$
\operatorname{dist}_{H}(A, B)=\sup _{a \in A} \inf _{b \in B}\|a-b\|_{H},
$$

where $\|\cdot\|_{H}=\|\cdot\|$ denotes the norm on $H$.

The global attractor for a discrete dynamical system induced by a map $S_{\text {discrete }}$ on a Banach space $H$ is defined in a similar fashion with the continuous time $t$ replaced by discrete time $n=0,1,2, \ldots$.

A dynamical system is called dissipative if it possesses a global attractor.

It is easy to see, thanks to the invariance and the attracting property, that the global attractor, when it exists, is unique [14, 32. We also caution the reader that our definition of dissipativity may be slightly different from the traditional notation [14, 32.

We are usually interested in $\int_{H} \Phi(\mathbf{u}) d \mu(\mathbf{u})$ (statistical average) for various test functionals $\Phi$. These test functionals are also called observable in the physics literature. We normally do not have direct access to any physically relevant invariant measure $\mu$ of the system a priori. One of the commonly used methods in calculating 
the statistical quantity is to substitute spatial average by long time average under Boltzmann's assumption of ergodicity $([12,21,23,37)$

$$
\int_{H} \Phi(\mathbf{u}) d \mu(\mathbf{u})=\lim _{t \rightarrow \infty} \frac{1}{t} \int_{0}^{t} \Phi(S(s) \mathbf{u}) d s .
$$

Although the above relationship is true for ergodic invariant measures and almost all initial data (with respect to the given ergodic invariant measure), the relationship is in general false for non-ergodic invariant measures since the long time average which exists for almost all initial data (with respect to the given invariant measure) may depend on the initial data and hence may not be a constant (the spatial average) ([21, 37]). One way to circumvent this difficulty is to replace the long time limit by Banach (generalized) limits ([22, section 4.2) which are bounded linear functionals on the space of bounded functions that agree with the usual long time limit on those functions whenever the long time limit exists. One may show via the socalled Bogliubov-Krylov argument that these generalized long time averages over a trajectory lead to invariant measures (which may depend on the chosen Banach limit and initial datum $\mathbf{u}$ ) of the system for appropriate dissipative dynamical systems, and the spatial and temporal averages are equivalent (see for instance [12, section 4.3 , or [40, Theorem 2).

Due to the presumed complexity of the dynamics, the physically interesting stationary statistical properties need to be calculated using numerical methods in the generic case. Even under the ergodicity assumption, it is not at all clear that classical numerical schemes which provide an accurate approximation on finite time intervals will remain meaningful for stationary statistical properties (long time properties) since small errors will be amplified and accumulated over long time except in the case that the underlying dynamics is asymptotically stable [13, 15, 20] where the statistical approach is not necessary since there is no chaos. Therefore, it is of great importance and a challenge to search for numerical methods that are able to capture stationary statistical properties of infinite-dimensional complex dynamical systems. We will focus on dissipative systems and time discretizations here since long time approximation seems to be the key issue involved.

As we shall demonstrate below, if the system and the scheme possess three very natural properties, then the stationary statistical properties of the scheme will converge to stationary statistical properties of the continuous dynamical system. The first property is the uniform dissipativity in the sense that the scheme possesses a global attractor for a small enough time step and the union of these global attractors (for different time steps) is pre-compact in the phase space. The second property is the uniform convergence of the numerical scheme for data from the support of the invariant measures on the unit time interval $[0,1]$ modulo an initial layer. The third property is that the continuous dynamical system is uniformly continuous (for data from the support of the invariant measures) on the unit time interval $[0,1]$. Our result will be presented in an abstract fashion in order to clarify the central issues and to provide well-organized means for discussing the problems. We also hope that our work will stimulate further work on accurate and efficient numerical schemes for stationary statistical properties of infinite-dimensional dissipative systems.

It is easy to see that the assumptions are natural. Since the underlying dynamical system is dissipative, it is natural to require that the numerical scheme inherit the dissipativity of the continuous-in-time system so that the scheme is uniformly dissipative (for small time steps). The uniform convergence of the numerical scheme 
for initial data from the global attractor on the unit time interval is also expected from most reasonable numerical schemes. The strong continuity of the underlying dynamical system on the unit time interval, uniform with respect to initial data from the union of the global attractors, is natural for most continuous dissipative dynamical systems. Once the desired natural conditions are discovered, the proof of the main result is relatively straightforward, although verifying these three sufficient conditions for each application may be highly non-trivial (see section 3 for a relatively easy application to the infinite Prandtl number model for convection with a linear semi-implicit scheme).

Although we are not aware of any work on the convergence of stationary statistical properties of numerical schemes for chaotic PDEs except our previous work [4, 5] (see [6] for the case of a map on the unit interval, and [26, 29, 35] for the case of Hamiltonian ODEs and more), there has been a lot of work on temporal approximation of dissipative dynamical systems such as the two-dimensional incompressible Navier-Stokes system and the one-dimensional Kuramoto-Sivashinsky equation (see [13, 16, 18, 27, 28, 33, 10, 11 among others). These authors were mostly interested in the long time stability of the scheme in the sense of deriving uniform in time bounds on the scheme (sometimes bound in the phase space $H$ only which is not sufficient for uniform dissipativity although it may be sufficient for the convergence of the global attractors), and none of them discussed statistical properties (except those works on Hamiltonian ODEs cited above). Our result on the convergence of stationary statistical properties may be viewed as an abstraction and generalization of 4, 5. See [9] for the heat bath approach to computing invariant measures for finite-dimensional systems and 2] for more on different sampling methods.

A by-product of the convergence analysis of the invariant measures presented here is the convergence of the global attractors of the scheme to that of the underlying system. This is also within expectation since the global attractors carry the support of the invariant measures. The convergence of the global attractors under time discretization has been discussed for the two-dimensional Navier-Stokes system, reaction-diffusion equation, and for finite-dimensional dynamical systems [27, 30, 16] among others. Therefore our result on the convergence of global attractors may be viewed as a generalization and abstraction of these results. However, we would like to point out that the convergence of the global attractors can be established under much weaker assumptions. One only needs the uniform boundedness of the union of the global attractors $K$, instead of the pre-compactness (plus finite time uniform convergence for data from $K$ ). Because of this important distinction, it is possible to have schemes that are able to capture the global attractor asymptotically but not necessarily the stationary statistical properties (invariant measures). There are also interesting works on persistence under approximation of various invariant sets (such as steady state, time periodic orbit, inertial manifold, etc.) both for PDEs and ODEs under appropriate assumptions (such as the spectral gap condition that is usually associated with inertial manifold theory, see [30, 17, 31, 32] and the references therein). We would also like to point out that the convergence of invariant sets and the convergence of stationary statistical properties are two related but very different issues associated with the long time behavior. It is easy to construct two dynamical systems with exactly the same global attractor or inertial manifold but with totally different dynamics or stationary statistical properties. 
The rest of the manuscript is organized as follows: in section 2 we prove the main results, namely the convergence of stationary statistical properties and the global attractors under the three natural hypotheses; in section 3 we discuss an application of the main results to the infinite Prandtl number model for convection. The choice of application is both for its physical significance and mathematical simplicity so that an essentially self-contained short exposition is possible and we believe that our main abstract theorem applies to many other dissipative systems and schemes. We then provide a conclusion and some remarks in the fourth/last section.

\section{Main Results: Abstract formulation}

Here we show our main results, namely, that uniform dissipativity plus finite time uniform convergence of the time discrete approximation together with the finite time uniform continuity of the underlying dynamical system imply convergence of the stationary statistical properties / invariant measures.

Throughout this section, all semigroups are assumed to be continuous in the sense that $S(t), t \geq 0$ and $S_{k}$ are continuous operators on $H$.

Theorem 1 (Convergence of Stationary Statistical Properties). Let $\{S(t), t \geq$ $0\}$ be a continuous semi-group on a separable Hilbert space $H$ which generates a continuous dissipative dynamical systems (in the sense of possessing a compact global attractor $\mathcal{A})$ on $H$. Let $\left\{S_{k}, 0<k \leq k_{0}\right\}$ be a family of continuous maps on $H$ that generates a family of discrete dissipative dynamical systems (with global attractor $\mathcal{A}_{k}$ ) on $H$. Suppose that the following three conditions are satisfied.

H1: (Uniform dissipativity) There exists a $k_{1} \in\left(0, k_{0}\right)$ such that $\left\{S_{k}, 0<k \leq\right.$ $\left.k_{1}\right\}$ is uniformly dissipative in the sense that

$$
K=\bigcup_{0<k \leq k_{1}} \mathcal{A}_{k}
$$

is pre-compact in $H$.

H2: (Uniform convergence on the unit time interval) $S_{k}$ uniformly converges to $S$ on the unit time interval (modulo an initial layer) and uniformly for initial data from the global attractor of $S_{k}$ in the sense that for any $t_{0} \in$ $(0,1)$,

$$
\lim _{k \rightarrow 0} \sup _{\mathbf{u} \in \mathcal{A}_{k}, n k \in\left[t_{0}, 1\right]}\left\|S_{k}^{n} \mathbf{u}-S(n k) \mathbf{u}\right\|=0 .
$$

H3: (Uniform continuity of the continuous system) $\{S(t), t \geq 0\}$ is uniformly continuous on $K$ on the unit time interval in the sense that for any $T^{*} \in$ $[0,1]$

$$
\lim _{t \rightarrow T^{*}} \sup _{\mathbf{u} \in K}\left\|S(t) \mathbf{u}-S\left(T^{*}\right) \mathbf{u}\right\|=0 .
$$

Then the invariant measures of the discrete dynamical system $\left\{S_{k}, 0<k \leq k_{0}\right\}$ converge to invariant measures of the continuous dynamical system $S$. More precisely, let $\mu_{k} \in \mathcal{I} \mathcal{M}_{k}$, where $\mathcal{I} \mathcal{M}_{k}$ denotes the set of all invariant measures of $S_{k}$. There must exist a subsequence, still denoted $\left\{\mu_{k}\right\}$, and $\mu \in \mathcal{I} \mathcal{M}$ (an invariant measure of $S(t))$, such that $\mu_{k}$ weakly converges to $\mu$, i.e.,

$$
\mu_{k} \rightarrow \mu \text {, as } k \rightarrow 0 \text {. }
$$


Moreover, extremal statistics converge in an upper-semi-continuous fashion in the sense that for any bounded continuous functional $\Phi$ on the phase space $H$, there exist ergodic invariant measures $\mu_{k} \in \mathcal{I M}_{k}$ and an ergodic invariant measure $\mu \in \mathcal{I} \mathcal{M}$, such that

$$
\begin{gathered}
\sup _{\mathbf{u}_{0} \in H} \limsup _{N \rightarrow \infty} \frac{1}{N} \sum_{n=1}^{N} \Phi\left(S_{k}^{n}\left(\mathbf{u}_{0}\right)\right)=\int_{H} \Phi(\mathbf{u}) d \mu_{k}(\mathbf{u}) \\
=\lim _{N \rightarrow \infty} \frac{1}{N} \sum_{n=1}^{N} \Phi\left(S_{k}^{n}\left(\mathbf{v}_{0}\right)\right) \text {, a.s. w.r.t. } \mu_{k}, \\
\sup _{\mathbf{u}_{0} \in H} \limsup _{T^{*} \rightarrow \infty} \frac{1}{T^{*}} \int_{0}^{T^{*}} \Phi\left(S(t) \mathbf{u}_{0}\right) d t=\int_{H} \Phi(\mathbf{u}) d \mu(\mathbf{u}) \\
=\lim _{T^{*} \rightarrow \infty} \frac{1}{T^{*}} \int_{0}^{T^{*}} \Phi\left(S(t) \mathbf{v}_{0}\right) d t, \text { a.s. w.r.t. } \mu, \\
\limsup _{k \rightarrow 0} \sup _{\mathbf{u}_{0} \in H} \limsup _{N \rightarrow \infty} \frac{1}{N} \sum_{n=1}^{N} \Phi\left(S_{k}^{n}\left(\mathbf{u}_{0}\right)\right) \\
\leq \sup _{\mathbf{u}_{0} \in H} \limsup _{T^{*} \rightarrow \infty} \frac{1}{T^{*}} \int_{0}^{T^{*}} \Phi\left(S(t) \mathbf{u}_{0}\right) d t .
\end{gathered}
$$

Proof. Since $K=\bigcup_{0<k<k_{1}} \mathcal{A}_{k}$ is pre-compact in $H$ and since all invariant measures are supported on the global attractor [12, 40] and $\mu_{k} \in \mathcal{I} \mathcal{M}_{k}$, we see that $\left\{\mu_{k}\right\}$ is tight in the space of all Borel probability measures on $H$ thanks to Prokhorov's theorem [1, 22, 12. Hence it must contain a weakly convergent subsequence (still denoted $\left\{\mu_{k}\right\}$ ) which weakly converges to a Borel probability measure $\mu$ on $H$; i.e.,

$$
\int_{H} \varphi(\mathbf{u}) d \mu_{k}(\mathbf{u}) \rightarrow \int_{H} \varphi(\mathbf{u}) d \mu(\mathbf{u}), \text { as } k \rightarrow 0
$$

for all bounded and continuous functionals $\varphi$ on $H$.

Our goal is to show that $\mu$ is invariant under $S(t)$.

Now we fix a $T^{*} \in(0,1]$ and let $n_{k}=\left\lfloor\frac{T^{*}}{k}\right\rfloor$ be the floor of $\frac{T^{*}}{k}$ (the largest integer dominated by $\left.\frac{T^{*}}{k}\right)$, and let $\varphi$ be any smooth $\left(C^{1}\right)$ test functional with compact support.

Since $\mu_{k} \in \mathcal{I} \mathcal{M}_{k}$, we have by the weak invariance of $\mu_{k}$ under $S_{k}$ and weak convergence,

$$
\int_{H} \varphi\left(S_{k}^{n_{k}} \mathbf{u}\right) d \mu_{k}(\mathbf{u})=\int_{H} \varphi(\mathbf{u}) d \mu_{k}(\mathbf{u}) \rightarrow \int_{H} \varphi(\mathbf{u}) d \mu(\mathbf{u}), \text { as } k \rightarrow 0 .
$$

On the other hand,

$$
\begin{aligned}
\int_{H} \varphi & \left(S_{k}^{n_{k}} \mathbf{u}\right) d \mu_{k}(\mathbf{u})=\int_{H} \varphi\left(S\left(T^{*}\right) \mathbf{u}\right) d \mu_{k}(\mathbf{u}) \\
& +\int_{H}\left(\varphi\left(S\left(n_{k} k\right) \mathbf{u}\right)-\varphi\left(S\left(T^{*}\right) \mathbf{u}\right)\right) d \mu_{k}(\mathbf{u}) \\
& +\int_{H}\left(\varphi\left(S_{k}^{n_{k}} \mathbf{u}\right)-\varphi\left(S\left(n_{k} k\right) \mathbf{u}\right)\right) d \mu_{k}(\mathbf{u})
\end{aligned}
$$


For the first term, since $S(t)$ is continuous, $\varphi\left(S\left(T^{*}\right) \mathbf{u}\right)$ is bounded and continuous in $\mathbf{u}$. Hence we have

$$
\int_{H} \varphi\left(S\left(T^{*}\right) \mathbf{u}\right) d \mu_{k}(\mathbf{u}) \rightarrow \int_{H} \varphi\left(S\left(T^{*}\right) \mathbf{u}\right) d \mu(\mathbf{u}), \text { as } k \rightarrow 0 .
$$

We also have, thanks to the uniform convergence assumption, the mean value theorem, and the uniform boundedness of the derivative of the smooth $\left(C^{1}\right)$ test functional with compact support, for $\mathbf{u} \in \mathcal{A}_{k}$,

$$
\begin{aligned}
\left|\varphi\left(S_{k}^{n_{k}} \mathbf{u}\right)-\varphi\left(S\left(n_{k} k\right) \mathbf{u}\right)\right| & \leq \sup _{\mathbf{u} \in H}\left\|\varphi^{\prime}(\mathbf{u})\right\| \sup _{\mathbf{u} \in \mathcal{A}_{k}}\left\|S_{k}^{n_{k}} \mathbf{u}-S\left(n_{k} k\right) \mathbf{u}\right\| \\
& \leq \sup _{\mathbf{u} \in H}\left\|\varphi^{\prime}(\mathbf{u})\right\| \sup _{\mathbf{u} \in \mathcal{A}_{k}, n k \in\left[\frac{T^{*}}{2}, T^{*}\right]}\left\|S_{k}^{n} \mathbf{u}-S(n k) \mathbf{u}\right\| \\
& \rightarrow 0, \text { as } k \rightarrow 0,
\end{aligned}
$$

and hence, since the support of the invariant measure $\mu_{k}$ is included in the global attractor $\mathcal{A}_{k}$, 12, 40,

$$
\int_{H}\left(\varphi\left(S_{k}^{n_{k}} \mathbf{u}\right)-\varphi\left(S\left(n_{k} k\right) \mathbf{u}\right)\right) d \mu_{k}(\mathbf{u}) \rightarrow 0, \text { as } k \rightarrow 0 .
$$

Moreover,

$\left|\varphi\left(S\left(n_{k} k\right) \mathbf{u}\right)-\varphi\left(S\left(T^{*}\right) \mathbf{u}\right)\right| \leq \sup _{\mathbf{u} \in H}\left\|\varphi^{\prime}(\mathbf{u})\right\| \sup _{\mathbf{u} \in \mathcal{A}_{k}}\left\|S\left(n_{k} k\right) \mathbf{u}-S\left(T^{*}\right) \mathbf{u}\right\| \rightarrow 0$, as $k \rightarrow 0$

by the uniform continuity of $S(t)$ at $t=T^{*}$ and $\mathbf{u} \in K$ since $n_{k} k=\left\lfloor\frac{T^{*}}{k}\right\rfloor k \rightarrow T^{*}$ as $k \rightarrow 0$. This further implies that

$$
\int_{H}\left(\varphi\left(S\left(n_{k} k\right) \mathbf{u}\right)-\varphi\left(S\left(T^{*}\right) \mathbf{u}\right)\right) d \mu_{k}(\mathbf{u}) \rightarrow 0, \text { as } k \rightarrow 0 .
$$

Combining the estimates above, we have

$$
\int_{H} \varphi\left(S_{k}^{n_{k}} \mathbf{u}\right) d \mu_{k}(\mathbf{u}) \rightarrow \int_{H} \varphi\left(S\left(T^{*}\right) \mathbf{u}\right) d \mu(\mathbf{u}), \text { as } k \rightarrow 0 .
$$

Therefore

$$
\int_{H} \varphi\left(S\left(T^{*}\right) \mathbf{u}\right) d \mu(\mathbf{u})=\int_{H} \varphi(\mathbf{u}) d \mu(\mathbf{u}),
$$

which is exactly the weak invariance (2) for the smooth $\left(C^{1}\right)$ test functional with compact support and $T^{*} \in(0,1]$.

For a general bounded continuous test functional $\varphi$, we can first approximate it by a finite-dimensional test functional of the form $\varphi \circ P_{m}$, where $P_{m}$ is the orthogonal projection onto the $m$-dimensional subspace spanned by the first $m$ elements of a given (fixed) orthonormal basis of $H$ (this is where we need $H$ to be a separable Hilbert space). We can then approximate $\varphi \circ P_{m}$ by smooth test functionals with compact support using mollifiers and truncation since only the value of $\varphi \circ P_{m}$ on the compact global attractor is relevant for statistics. This proves short time weak invariance (2) for any bounded continuous test functional $\varphi$ and $T^{*} \in(0,1]$. 
Now for a general $T^{* *}>1$, there exists a unique positive integer $n$ and $T_{*} \in(0,1]$ such that $T^{* *}=n+T_{*}$. Hence

$$
\begin{aligned}
\int_{H} \varphi\left(S\left(T^{* *}\right) \mathbf{u}\right) d \mu(\mathbf{u}) & =\int_{H} \varphi\left(S^{n}(1) S\left(T_{*}\right) \mathbf{u}\right) d \mu(\mathbf{u}) \\
& =\int_{H} \varphi\left(S\left(T_{*}\right) \mathbf{u}\right) d \mu(\mathbf{u}) \\
& =\int_{H} \varphi(\mathbf{u}) d \mu(\mathbf{u}),
\end{aligned}
$$

where we have utilized the semi-group property of $S(t)$, the strong continuity of $S(t)$ and the short time weak invariance that we proved above with $T^{*}=1 n$ times and $T^{*}=T_{*}$ one time.

This ends the proof of the convergence of the invariant measures.

The first half of the results on the extremal statistics is a consequence of the fact that extremal statistics are saturated by ergodic invariant measures (see for instance [37] or [40, Theorem 5]). We sketch the proof for convenience. Recall that for any fixed bounded continuous test functional $\Phi$ and initial data $\mathbf{u}_{0}$, it is possible to choose a special Banach limit LIM which agrees with the limsup on the orbit [22, 40, and hence there exist invariant measures $\mu_{k, \mathbf{u}_{0}} \in \mathcal{I M}_{k}, \mu_{\mathbf{u}_{0}} \in \mathcal{I} \mathcal{M}$ such that

$$
\begin{aligned}
L I M_{N \rightarrow \infty} \frac{1}{N} \sum_{n=1}^{N} \Phi\left(S_{k}^{n}\left(\mathbf{u}_{0}\right)\right) & =\limsup _{N \rightarrow \infty} \frac{1}{N} \sum_{n=1}^{N} \Phi\left(S_{k}^{n}\left(\mathbf{u}_{0}\right)\right)=\int_{H} \Phi(\mathbf{u}) d \mu_{k, \mathbf{u}_{0}}(\mathbf{u}), \\
L I M_{T^{*} \rightarrow \infty} \frac{1}{T^{*}} \int_{0}^{T^{*}} \Phi\left(S(t) \mathbf{u}_{0}\right) d t & =\limsup _{T^{*} \rightarrow \infty} \frac{1}{T^{*}} \int_{0}^{T^{*}} \Phi\left(S(t) \mathbf{u}_{0}\right) d t \\
& =\int_{H} \Phi(\mathbf{u}) d \mu_{\mathbf{u}_{0}}(\mathbf{u}) .
\end{aligned}
$$

On the other hand, extremal points of the set of invariant measures are ergodic (37] or [40, Theorem 3]) and hence there exist ergodic invariant measures $\mu_{k} \in$ $\mathcal{I M}_{k}, \mu \in \mathcal{I} \mathcal{M}$ such that

$$
\begin{aligned}
\sup _{\nu_{k} \in \mathcal{I} \mathcal{M}_{k}} \int_{H} \Phi(\mathbf{u}) d \nu_{k}(\mathbf{u}) & =\int_{H} \Phi(\mathbf{u}) d \mu_{k}(\mathbf{u})=\lim _{N \rightarrow \infty} \frac{1}{N} \sum_{n=1}^{N} \Phi\left(S_{k}^{n}\left(\mathbf{u}_{0}\right)\right), \text { a.s. w.r.t. } \mu_{k}, \\
\sup _{\nu \in \mathcal{I} \mathcal{M}} \int_{H} \Phi(\mathbf{u}) d \nu(\mathbf{u}) & =\int_{H} \Phi(\mathbf{u}) d \mu(\mathbf{u})=\lim _{T^{*} \rightarrow \infty} \frac{1}{T^{*}} \int_{0}^{T^{*}} \Phi\left(S(t) \mathbf{u}_{0}\right) d t, \text { a.s. w.r.t. } \mu,
\end{aligned}
$$

Combining the above two sets of equations together with the tightness of $\mathcal{I} \mathcal{M}_{k}, \mathcal{I M}$, we arrive at (10, 11).

As for the upper semi-convergence of the extremal statistics stated in (12), we have, thanks to the uniform dissipativity and Prokhorov's theorem, that there exists a subsequence (still denoted $\left\{\mu_{k}\right\}$ ) and $\nu \in \mathcal{I} \mathcal{M}$ such that

$$
\begin{aligned}
\mu_{k} & \rightarrow \nu, \text { as } k \rightarrow 0 \\
\limsup _{k \rightarrow 0} \sup _{\mathbf{u}_{0} \in H} \limsup _{N \rightarrow \infty} \frac{1}{N} \sum_{n=1}^{N} \Phi\left(S_{k}^{n}\left(\mathbf{u}_{0}\right)\right) & =\limsup _{k \rightarrow 0} \int_{H} \Phi(\mathbf{u}) d \mu_{k} .
\end{aligned}
$$


Since $\mathcal{I M}$ is compact in the space of Borel probability measures on $H$, there exists an ergodic invariant measure $\nu_{\max } \in \mathcal{I} \mathcal{M}$ such that $\sup _{\tilde{\mu} \in \mathcal{I} \mathcal{M}} \int_{H} \Phi(\mathbf{u}) d \tilde{\mu}=$ $\int_{H} \Phi(\mathbf{u}) d \nu_{\max }$ [40]. Therefore,

$$
\begin{aligned}
\limsup _{k \rightarrow 0} \sup _{\mathbf{u}_{0} \in H} \limsup _{N \rightarrow \infty} \frac{1}{N} \sum_{n=1}^{N} \Phi\left(S_{k}^{n}\left(\mathbf{u}_{0}\right)\right) & =\limsup _{k \rightarrow 0} \int_{H} \Phi(\mathbf{u}) d \mu_{k} \\
& =\int_{H} \Phi(\mathbf{u}) d \nu \\
& \leq \sup _{\tilde{\mu} \in \mathcal{I} \mathcal{M}} \int_{H} \Phi(\mathbf{u}) d \tilde{\mu} \\
& =\int_{H} \Phi(\mathbf{u}) d \nu_{\max } \\
& =\lim _{T^{*} \rightarrow \infty} \frac{1}{T^{*}} \int_{0}^{T^{*}} \Phi\left(S(t) \mathbf{u}_{0}\right) d t \\
& \leq \sup _{\mathbf{u}_{0} \in H} \lim _{T^{*} \rightarrow \infty} \frac{1}{T^{*}} \int_{0}^{T^{*}} \Phi\left(S(t) \mathbf{u}_{0}\right) d t .
\end{aligned}
$$

This completes the proof of the theorem.

Next, we show the convergence of the global attractors under weaker assumptions, namely the uniform boundedness of $K$ (the union of the global attractors), and uniform convergence on a finite time interval (modulo an arbitrary initial layer).

Proposition 1 (Convergence of Global Attractors). Let $\{S(t), t \geq 0\}$ be a continuous semi-group on a Banach space $H$ that generates a dissipative dynamical system (in the sense of possessing a compact global attractor $\mathcal{A}$ ) on $H$. Let $\left\{S_{k}, 0<k \leq k_{0}\right\}$ be a family of continuous maps on $H$ that generates a family of discrete dissipative dynamical systems (with global attractor $\mathcal{A}_{k}$ ) on $H$. Suppose that the following two conditions are satisfied.

H4: (Uniform boundedness) There exists a $k_{1} \in\left(0, k_{0}\right]$ such that $\left\{S_{k}, 0<k \leq\right.$ $\left.k_{1}\right\}$ is uniformly bounded in the sense that

$$
K=\bigcup_{0<k \leq k_{1}} \mathcal{A}_{k}
$$

is bounded in $H$.

H5: (Finite time uniform convergence) $S_{k}$ uniformly converges to $S$ on any finite time interval (modulo any initial layer) and uniformly for initial data from the global attractor of the scheme in the sense that there exists $t_{0}>0$ such that for any $T^{*}>t_{0}>0$,

$$
\lim _{k \rightarrow 0} \sup _{\mathbf{u} \in \mathcal{A}_{k}, n k \in\left[t_{0}, T^{*}\right]}\left\|S_{k}^{n} \mathbf{u}-S(n k) \mathbf{u}\right\|=0 .
$$

Then the global attractors converge in the sense of Hausdorff semi-distance, i.e.

$$
\lim _{k \rightarrow 0} \operatorname{dist}_{H}\left(\mathcal{A}_{k}, \mathcal{A}\right)=0 \text {. }
$$


Proof. Since $K$ is bounded, for any given $\epsilon>0$, there exists a $T_{\epsilon}>t_{0}>0$ such that

$$
\operatorname{dist}_{H}(S(t) K, \mathcal{A})<\frac{\epsilon}{2}, \forall t \geq T_{\epsilon}
$$

because the global attractor $\mathcal{A}$ attracts all bounded sets, in particular $K$.

Now let $\mathbf{u}_{k} \in \mathcal{A}_{k}$. Since the global attractor $\mathcal{A}_{k}$ is invariant under $S_{k}$, there exists a $\mathbf{v}_{k} \in \mathcal{A}_{k}$ such that $\mathbf{u}_{k}=S_{k}^{n_{k}} \mathbf{v}_{k}$, where $n_{k}=\left\lfloor\frac{T_{\epsilon}+1}{k}\right\rfloor$.

Thanks to the uniform convergence on $\left[T_{\epsilon}, T_{\epsilon}+1\right]$ and $\mathbf{u} \in K$, there exists $k_{\epsilon}>0$ such that

$$
\left\|\mathbf{u}_{k}-S\left(n_{k} k\right) \mathbf{v}_{k}\right\|=\left\|S_{k}^{n_{k}} \mathbf{v}_{k}-S\left(n_{k} k\right) \mathbf{v}_{k}\right\|<\frac{\epsilon}{2}, k \leq k_{\epsilon} .
$$

This implies that

$$
\begin{aligned}
\operatorname{dist}_{H}\left(\mathcal{A}_{k}, \mathcal{A}\right) & =\sup _{\mathbf{u}_{k} \in \mathcal{A}_{k}} \operatorname{dist}\left(\mathbf{u}_{k}, \mathcal{A}\right) \\
& \leq \sup _{\mathbf{u}_{k} \in \mathcal{A}_{k}}\left(\left\|\mathbf{u}_{k}-S\left(n_{k} k\right) \mathbf{v}_{k}\right\|+\operatorname{dist}\left(S\left(n_{k} k\right) \mathbf{v}_{k}, \mathcal{A}\right)\right) \\
& \leq \epsilon, k \leq k_{\epsilon} .
\end{aligned}
$$

This completes the proof for the convergence of the global attractors.

We would like to reiterate the point than the uniform boundedness assumption H4 is much weaker than the uniform dissipativity assumption H1 for infinitedimensional systems although they are equivalent for finite-dimensional systems. This is an important difference and hence it is theoretically possible to have schemes that are able to capture the global attractor asymptotically but not the invariant measures necessarily for infinite-dimensional systems. Conditions H5 and $\mathrm{H} 2$ are almost the same. H2 is slightly stronger than $\mathrm{H} 5$ in some sense since $\mathrm{H} 2$ requires the uniform convergence of the scheme on $\left[t_{0}, 1\right], \forall t_{0} \in(0,1)$ while $\mathrm{H} 5$ only requires the uniform convergence of the scheme on $\left[t_{0}, T^{*}\right]\left(T^{*} \geq 1\right)$ with one $t_{0} \in(0,1)$. On the other hand, $\mathrm{H} 5$ is slightly stronger than $\mathrm{H} 2$ in some other sense since only $T^{*}=1$ is needed in H2. They are usually valid for reasonable numerical schemes (see below for an example on the infinite Prandtl number model). Therefore convergence of the global attractors is usually easier to establish than the convergence of the invariant measures (stationary statistical properties). Related results on convergence of global attractors can be found in [14, 30, 25, among others.

In applications, the discrete dynamical systems $\left\{S_{k}\right\}$ are usually generated by one time step discretization (numerical scheme) with time step $k$. In other words, $\mathbf{u}^{n+1}=S_{k}\left(\mathbf{u}^{n}\right)$ is the solution to the numerical scheme. The uniform dissipativity of the numerical scheme is customarily established via the existence of a uniform (in time step) absorbing ball in another separable Hilbert space $V$ which is compactly imbedded in $H$ in the case of a strongly dissipative system (see the next section for an example). However, this may not be feasible for weakly dissipative systems such as the Darcy-Boussinesq system for convection in a fluid saturated porous media, or a weakly damped driven Schrödinger equation. The finite time uniform convergence comes with classical numerical analysis for reasonable schemes (see next section for an example). The uniform continuity of the underlying continuous dynamical system is also easily verified for reasonable systems. 


\section{Application to the infinite Prandtl number model for Convection}

Here we illustrate an application of the main result to the following infinite Prandtl number model for convection [3, 34, 7, 38, 8,

$$
\begin{aligned}
\nabla p & =\Delta \mathbf{u}+R a \mathbf{k} T, \quad \nabla \cdot \mathbf{u}=0,\left.\quad \mathbf{u}\right|_{z=0,1}=0, \\
\frac{\partial T}{\partial t}+\mathbf{u} \cdot \nabla T & =\Delta T,\left.\quad T\right|_{z=0}=1,\left.T\right|_{z=1}=0,
\end{aligned}
$$

where $\mathbf{u}$ is the Eulerian velocity of the fluid, $p$ represents the kinematic pressure of the fluid, $T$ is the temperature of the fluid, $\mathbf{k}$ is a unit vector in the $z$ direction, $R a$ is the Rayleigh number measuring the ratio of differential heating over overall dissipation, and we assume that the fluids occupy the (non-dimensionalized) region $\Omega=\left[0, L_{x}\right] \times\left[0, L_{y}\right] \times[0,1]$ with periodicity imposed in the horizontal directions for simplicity.

The choice of this example is both for its physical significance and for its mathematical simplicity so that we may have an essentially self-contained short exposition. It is well known that this system possesses turbulent behavior at large Rayleigh numbers [3, 7, 19, 34. The convergence of the stationary statistical properties for another class of semi-implicit schemes was discussed in [4, 5] utilizing a Liouville-type equation approach. The scheme that we study here has an added advantage over the schemes that we investigated earlier in the sense that the discretization is independent of any ad-hoc background temperature profile $\tau$ (to be introduced in (22) ).

Since the temperature field $T$ satisfies inhomogeneous boundary conditions, it is mathematically convenient to consider a perturbative temperature field

$$
\theta=T-\tau(z), \quad \tau(0)=1, \tau(1)=0,
$$

where $\tau(z)$ is a smooth fixed background temperature profile. This decomposition is also in accordance with the mean $(\tau)$ and fluctuation $(\theta)$ decomposition commonly used in the study of turbulent flows.

One of the most important statistical quantities in convection is the Nusselt number [3, 7, 8, 19, 39] quantifying the heat transport in the vertical direction, which is the statistics corresponding to the functional

$$
\Phi(\theta)=R a \int_{\Omega} A^{-1}(\mathbf{k} \theta)_{3} \theta d \mathbf{x}=\int_{\Omega} u_{3} \theta d \mathbf{x},
$$

where $A$ denotes the Stokes operator with the associated boundary conditions and viscosity one, and $A^{-1}(\mathbf{k} \theta)_{3}$ represents the third component (vertical velocity) of $A^{-1}(\mathbf{k} \theta)$. In other words, $\mathbf{u}=A^{-1} \mathbf{f}$ solves the following Stokes system:

$$
\begin{aligned}
-\Delta \mathbf{u}+\nabla p & =\mathbf{f}, & & \nabla \cdot \mathbf{u}=0, \\
\left.\mathbf{u}\right|_{z=0,1} & =0, & & \mathbf{u} \text { periodic in } x, y .
\end{aligned}
$$

Although this $\Phi$ is not bounded, for any pre-compact set $K$ (in particular the union of the global attractors of the scheme), we can easily replace it by a bounded continuous functional $\tilde{\Phi}$ which agrees with $\Phi$ on $K$ and hence the statistical averages of $\Phi$ and $\tilde{\Phi}$ are the same since the support of all interesting invariant measures is included in $K$. Our result on the convergence of the maximal Nusselt number, i.e. (12) with the $\Phi$ given above, complements the well-known variational approach [7] since our result indicates that the numerics will provide an asymptotic lower bound 
for the maximal Nusselt number while the variational approach provides a rigorous upper bound.

3.1. A semi-implicit scheme. Notice that the infinite Prandtl number model can be written as a non-local non-linear advection diffusion equation for the temperature field

$$
\frac{\partial T}{\partial t}+R a A^{-1}(\mathbf{k} T) \cdot \nabla T=\Delta T,\left.\quad T\right|_{z=0}=1,\left.T\right|_{z=1}=0,
$$

where $A$ denotes the Stokes operator with the associated boundary conditions and viscosity one. The system can also be written in terms of the perturbative temperature field $\theta$ as

$$
\frac{\partial \theta}{\partial t}+R a A^{-1}(\mathbf{k} \theta) \cdot \nabla \theta+R a A^{-1}(\mathbf{k} \theta)_{3} \tau^{\prime}(z)=\Delta \theta+\tau^{\prime \prime}(z) .
$$

We then propose the following semi-implicit scheme for the infinite Prandtl number model

$$
\frac{T_{k}^{n+1}-T_{k}^{n}}{k}+R a A^{-1}\left(\mathbf{k} T_{k}^{n}\right) \cdot \nabla T_{k}^{n+1}=\Delta T_{k}^{n+1},
$$

where $T_{k}^{n}$ denotes the approximate solution at time $k n$ with $k$ being the time step.

Our goal now is to show that the stationary statistical properties of this scheme converge to those of the infinite Prandtl number model as the time step $k$ approaches zero.

In order to accomplish this goal utilizing our main result proved in the previous section, we rewrite the scheme in the perturbative variable $\theta$ as usual so that the phase space is the Hilbert space $H=L^{2}(\Omega)$,

$$
\frac{\theta_{k}^{n+1}-\theta_{k}^{n}}{k}+R a A^{-1}\left(\mathbf{k} \theta_{k}^{n}\right) \cdot \nabla \theta_{k}^{n+1}+R a A^{-1}\left(\mathbf{k} \theta_{k}^{n}\right)_{3} \tau^{\prime}(z)=\Delta \theta_{k}^{n+1}+\tau^{\prime \prime}(z),
$$

where $\theta_{k}^{n}=T_{k}^{n}-\tau$ with $\tau(z)$ being an ad-hoc background temperature profile (to be specified below) which satisfies the non-homogeneous Dirichlet boundary condition of $T_{k}^{n}$, and we are searching for a solution in the space $H_{0, p e r}^{1}$ (the subspace of $H^{1}$ with zero trace/value at $z=0,1$ and periodic in the horizontal directions). Here $A^{-1}(\mathbf{k} \theta)_{3}$ represents the third component (vertical velocity) of $A^{-1}(\mathbf{k} \theta)$.

Following [7, we set the background temperature profile $\tau$ to be a locally smoothed (mollified) version of the following piecewise linear function:

$$
\tau(z)=\left\{\begin{array}{cc}
1-\frac{z}{\delta}, & 0 \leq z \leq \delta \\
0, & \delta \leq z \leq 1
\end{array}\right.
$$

The choice of the parameter $\delta$ will be specified later in (26).

We remark that the scheme (21) is different from the scheme that we investigated earlier [4, 5], where the scheme

$$
\frac{\theta_{k}^{n+1}-\theta_{k}^{n}}{k}+R a A^{-1}\left(\mathbf{k} \theta_{k}^{n}\right) \cdot \nabla \theta_{k}^{n+1}+R a A^{-1}\left(\mathbf{k} \theta_{k}^{n+1}\right){ }_{3} \tau^{\prime}(z)=\Delta \theta_{k}^{n+1}+\tau^{\prime \prime}(z)
$$

was studied. The new scheme (21) has the advantage that it is independent of the ad-hoc background profile $\tau$ since it can be written in terms of the temperature field $T$ only as is clear in (20). 
It is easy to see that the well-posedness of the scheme (21) follows from the weak formulation

$$
\begin{aligned}
\int_{\Omega}\left(\frac{1}{k} \theta_{k}^{n+1}+R a A^{-1}\left(\mathbf{k} \theta_{k}^{n}\right) \cdot \nabla \theta_{k}^{n+1}\right) \psi+\int_{\Omega} \nabla \theta_{k}^{n+1} \cdot \nabla \psi \\
=\int_{\Omega}\left(\frac{1}{k} \theta_{k}^{n}+\tau^{\prime \prime}-R a A^{-1}\left(\mathbf{k} \theta_{k}^{n}\right)_{3} \tau^{\prime}(z)\right) \psi, \forall \psi \in H_{0, p e r}^{1},
\end{aligned}
$$

the identity $\int_{\Omega} A^{-1}\left(\mathbf{k} \theta_{k}^{n}\right) \cdot \nabla \theta_{k}^{n+1} \theta_{k}^{n+1}=0, \forall \theta_{k}^{n+1} \in H_{0, p e r}^{1}, \theta_{k}^{n} \in L^{2}$, and the LaxMilgram theorem 22]. Therefore, we have a discrete dynamical system $S_{k}$ defined on the Hilbert space

$$
H=L^{2}(\Omega)
$$

given by

$$
S_{k}\left(\theta_{k}^{n}\right)=\theta_{k}^{n+1}
$$

We notice that $S_{k}$ in fact maps $H$ into $H_{0, p e r}^{1}$, which is a direct consequence of the weak formulation.

In order to apply the main result proved in the previous theorem to the current situation, we will verify the three conditions postulated in the main theorem. These will be the goal of the next three subsections.

Our main result is the following theorem on the semi-implicit scheme (21) for the infinite Prandtl number model for convection.

Theorem 2. The semi-implicit linear scheme (21) with time step $k$ defines a dissipative discrete dynamical system $S_{k}$ on the phase space $H=L^{2}(\Omega)$. The stationary statistical properties as well as the global attractor of this scheme (discrete dynamical system) converges to those of the infinite Prandtl number model for convection (18) in the form specified in Theorem 1 and Proposition 1.

3.2. Uniform dissipativity. Here we demonstrate the uniform dissipativity, i.e., H1, of the scheme (21) with an appropriate choice of $\delta$.

Here and below, the $c_{j}$ s denote generic constants independent of $k, n$ and the initial data unless otherwise stated (but which may depend on the Rayleigh number). Here and elsewhere $\|\theta\|=\sqrt{\int_{\Omega}|\theta|^{2}}$ denotes the spatial $L^{2}$ norm of $\theta$, and

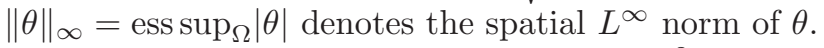

We first derive a uniform bound in the $L^{2}$ space. For this purpose we take the inner product of the scheme (21) with $\psi=\theta_{k}^{n+1}$ and utilize the identity $(a-b, a)=$ $\frac{1}{2}\left(|a|^{2}-|b|^{2}+|a-b|^{2}\right)$, and we have

$$
\begin{aligned}
& \frac{1}{2 k}\left(\left\|\theta_{k}^{n+1}\right\|^{2}-\left\|\theta_{k}^{n}\right\|^{2}+\left\|\theta_{k}^{n+1}-\theta_{k}^{n}\right\|^{2}\right)+\left\|\nabla \theta_{k}^{n+1}\right\|^{2} \\
& \quad \leq\left\|\tau^{\prime}\right\|\left\|\nabla \theta_{k}^{n+1}\right\|+R a\left|\int_{\Omega} \tau^{\prime}(z)\left(A^{-1}\left(\mathbf{k} \theta_{k}^{n}\right)\right)_{3} \theta_{k}^{n+1}\right| \\
& \quad \leq\left\|\tau^{\prime}\right\|^{2}+\frac{1}{4}\left\|\nabla \theta_{k}^{n+1}\right\|^{2}+R a\left|\int_{\Omega} \tau^{\prime}(z)\left(A^{-1}\left(\mathbf{k} \theta_{k}^{n}\right)\right)_{3} \theta_{k}^{n+1}\right|,
\end{aligned}
$$

where we have used the identity $\int_{\Omega} A^{-1}\left(\mathbf{k} \theta_{k}^{n}\right) \cdot \nabla \theta_{k}^{n+1} \theta_{k}^{n+1}=0$ one more time. 
As for the non-linear term, we have

$$
\begin{aligned}
R a\left|\int_{\Omega} \tau^{\prime}(z)\left(A^{-1}\left(\mathbf{k} \theta_{k}^{n}\right)\right)_{3} \theta_{k}^{n+1}\right| & \leq \frac{R a}{\delta}\left\|A^{-1}\left(\mathbf{k} \theta_{k}^{n}\right)_{3}\right\|_{L^{2}(z \leq \delta)}\left\|\theta_{k}^{n+1}\right\|_{L^{2}(z \leq \delta)} \\
& \leq R a \delta\left\|\frac{\partial}{\partial z} A^{-1}\left(\mathbf{k} \theta_{k}^{n}\right)_{3}\right\|_{L^{2}(z \leq \delta)}\left\|\frac{\partial}{\partial z} \theta_{k}^{n+1}\right\|_{L^{2}(z \leq \delta)} \\
& \leq R a \delta^{2}\left\|\frac{\partial^{2}}{\partial z^{2}} A^{-1}\left(\mathbf{k} \theta_{k}^{n}\right)_{3}\right\|_{L^{2}(z \leq \delta)}\left\|\frac{\partial}{\partial z} \theta_{k}^{n+1}\right\|_{L^{2}(z \leq \delta)} \\
& \leq c_{1} R a \delta^{2}\left\|\theta_{k}^{n}\right\|\left\|\frac{\partial}{\partial z} \theta_{k}^{n+1}\right\| \\
& \leq c_{1} R a \delta^{2}\left(\left\|\theta_{k}^{n}-\theta_{k}^{n+1}\right\|+\left\|\theta_{k}^{n+1}\right\|\right)\left\|\nabla \theta_{k}^{n+1}\right\| \\
& \leq c_{1} R a \delta^{2}\left\|\theta_{k}^{n}-\theta_{k}^{n+1}\right\|\left\|\nabla \theta_{k}^{n+1}\right\|+c_{1} R a \delta^{2}\left\|\nabla \theta_{k}^{n+1}\right\|^{2} \\
& \leq \frac{c_{1} R a \delta^{2}}{4}\left\|\theta_{k}^{n}-\theta_{k}^{n+1}\right\|^{2}+2 c_{1} R a \delta^{2}\left\|\nabla \theta_{k}^{n+1}\right\|^{2} \\
& \leq \frac{1}{4 k}\left\|\theta_{k}^{n}-\theta_{k}^{n+1}\right\|^{2}+\frac{1}{4}\left\|\nabla \theta_{k}^{n+1}\right\|^{2}
\end{aligned}
$$

provided that we choose the background temperature profile (through $\delta$ ) and the time step $k$ in the following fashion:

$$
\delta=\left(8 c_{1} R a\right)^{-\frac{1}{2}}, \quad k \leq 8,
$$

where we have utilized the specific form of the background profile $\tau$ (22), the homogeneous boundary conditions for $\theta_{k}^{n}, \theta_{k}^{n+1}, A^{-1}\left(\mathbf{k} \theta_{k}^{n}\right)_{3}, \frac{\partial}{\partial z} A^{-1}\left(\mathbf{k} \theta^{n}\right)_{3}$, elliptic regularity (for the Stokes operator $A$ ) and the Poincaré inequality.

Therefore, under the specific choice of the background temperature profile 22 . 26), there exists a constant $c_{2}$ such that

$$
\frac{1}{k}\left(\left\|\theta_{k}^{n+1}\right\|^{2}-\left\|\theta_{k}^{n}\right\|^{2}+\frac{1}{2}\left\|\theta_{k}^{n+1}-\theta_{k}^{n}\right\|^{2}\right)+\left\|\nabla \theta_{k}^{n+1}\right\|^{2} \leq 2\left\|\tau^{\prime}\right\|^{2} \leq c_{2} R a^{\frac{1}{2}},
$$

which further implies, thanks to the Poincaré inequality,

$$
(1+k)\left\|\theta_{k}^{n+1}\right\|^{2} \leq\left\|\theta_{k}^{n}\right\|^{2}+c_{2} k R a^{\frac{1}{2}} .
$$

This leads to, with the help of a simple iteration,

$$
\left\|\theta_{k}^{n+1}\right\|^{2} \leq(1+k)^{-(n+1)}\left\|\theta_{0}\right\|^{2}+c_{2} R a^{\frac{1}{2}} .
$$

This is a uniform estimate in the $L^{2}$ space since for any $\theta_{0} \in B_{R}\left(L^{2}\right)$ (ball centered at the origin with radius $R$ in $\left.L^{2}\right)$, there exists an integer $N_{0}(R, k)$ such that

$$
(1+k)^{-n}\left\|\theta_{0}\right\|^{2} \leq c_{2} R a^{\frac{1}{2}}, \forall n \geq N_{0}
$$

Hence

$$
\left\|\theta_{k}^{n}\right\| \leq \sqrt{2 c_{2}} R a^{\frac{1}{4}}, \forall n \geq N_{0}(R, k) .
$$

Indeed, we have

$$
N_{0}(R, k) \approx \frac{\ln \frac{R^{2}}{c_{2} R a^{\frac{1}{2}}}}{\ln (1+k)} \approx \frac{\ln \frac{R^{2}}{c_{2} R a^{\frac{1}{2}}}}{k}
$$

so that the time needed to enter the absorbing ball of radius $\sqrt{2 c_{2}} R a^{\frac{1}{4}}$ is roughly

$$
k N_{0}(R, k) \approx \ln \frac{R^{2}}{c_{2} R a^{\frac{1}{2}}},
$$

which is independentof $k, n$. 
There are two byproducts of the inequality (27). Namely, for any positive integer $N$, we have

$$
\begin{aligned}
& \frac{1}{N} \sum_{n=0}^{N}\left\|\nabla \theta_{k}^{n+1}\right\|^{2} \leq \frac{\left\|\theta_{0}\right\|^{2}}{k N}+c_{2} R a^{\frac{1}{2}} \\
& \sum_{n=0}^{N}\left\|\theta_{k}^{n+1}-\theta_{k}^{n}\right\|^{2} \leq 2\left\|\theta_{0}\right\|^{2}+2 c_{2} k N R a^{\frac{1}{2}} .
\end{aligned}
$$

Thanks to the Poincaré inequality, $\|\nabla \theta\|$ is equivalent to $\|\theta\|_{H^{1}}$ and hence the first inequality is a bound in $L^{2}\left(0, T^{*} ; H^{1}\right)$ for the scheme for any $T^{*}>0$. It is easy to see that the second is a bound on the difference of the solution at adjacent time steps.

Our next immediate goal is to obtain uniform estimates in $H^{1}$ for the solution of the scheme (21). The uniform in $H^{1}$ estimate will guarantee the uniform dissipativity (H1) since boundedness in $H^{1}$ implies pre-compactness in $L^{2}$ by the Rellich compactness theorem. For this purpose, we take the inner product of the scheme with $\psi=-\Delta \theta_{k}^{n+1}$ and we have

$$
\begin{aligned}
\frac{1}{2 k}(\| \nabla & \left.\theta_{k}^{n+1}\left\|^{2}-\right\| \nabla \theta_{k}^{n}\left\|^{2}+\right\| \nabla\left(\theta_{k}^{n+1}-\theta_{k}^{n}\right) \|^{2}\right)+\left\|\Delta \theta_{k}^{n+1}\right\|^{2} \\
\leq & \left\|\tau^{\prime \prime}\right\|\left\|\Delta \theta_{k}^{n+1}\right\|+R a\left\|\tau^{\prime}\right\|\left\|A^{-1}\left(\mathbf{k} \theta_{k}^{n}\right)\right\|_{\infty}\left\|\Delta \theta_{k}^{n+1}\right\| \\
& +R a\left\|A^{-1}\left(\mathbf{k} \theta_{k}^{n}\right)\right\|_{\infty}\left\|\nabla \theta_{k}^{n+1}\right\|\left\|\Delta \theta_{k}^{n+1}\right\| \\
\leq & \left\|\tau^{\prime \prime}\right\|\left\|\Delta \theta_{k}^{n+1}\right\|+c_{3} R a\left(\left\|\tau^{\prime}\right\|\left\|\theta_{k}^{n}\right\|\left\|\Delta \theta_{k}^{n+1}\right\|+\left\|\theta_{k}^{n}\right\|\left\|\theta_{k}^{n+1}\right\|^{\frac{1}{2}}\left\|\Delta \theta_{k}^{n+1}\right\|^{\frac{3}{2}}\right) \\
\leq & \left.c_{4}\left(1+(1+k)^{-n}\left\|\theta_{0}\right\|^{2}\right)^{\frac{1}{2}}\left\|\Delta \theta_{k}^{n+1}\right\|+c_{5}\left(1+(1+k)^{-n}\left\|\theta_{0}\right\|^{2}\right)^{\frac{3}{2}}\left\|\Delta \theta_{k}^{n+1}\right\|^{\frac{3}{2}}\right) \\
\leq & \frac{1}{2}\left\|\Delta \theta_{k}^{n+1}\right\|^{2}+c_{6}\left(1+(1+k)^{-n}\left\|\theta_{0}\right\|^{2}\right)^{6}
\end{aligned}
$$

where we have applied the regularity result for the Stokes operator $A$, the Sobolev imbedding of $H^{2}$ into $L^{\infty}$, an interpolation inequality, the uniform $L^{2}$ estimate (29) and a Hölder-type inequality.

This implies, when combined with (30, 33), that there exists $N_{1}(R, k) \geq N_{0}(R, k)$, and $c_{7} \geq 2 c_{2} R a^{\frac{1}{2}}$, such that

$$
\begin{aligned}
\left\|\nabla \theta_{k}^{n+1}\right\|^{2}-\left\|\nabla \theta_{k}^{n}\right\|^{2}+\left\|\nabla\left(\theta_{k}^{n+1}-\theta_{k}^{n}\right)\right\|^{2}+k\left\|\Delta \theta_{k}^{n+1}\right\|^{2} & \leq k c_{7}, \forall n \geq N_{1}, \\
\left\|\nabla \theta_{k}^{N_{1}}\right\|^{2} & \leq c_{7}
\end{aligned}
$$

where $N_{1}$ can be estimated as

$$
N_{1}(R, k) \approx N_{0}+\frac{2}{k}
$$

This implies the following uniform $H^{1}$ estimate with initial data from $B_{R}\left(L^{2}\right)$ :

$$
\left\|\nabla \theta_{k}^{n+1}\right\|^{2} \leq c_{7}, \forall n \geq N_{1} .
$$

This further leads to the existence of an absorbing ball in $H^{1}$ of radius $\sqrt{c_{7}}$ such that trajectories starting from $B_{R}\left(L^{2}\right)$ enter this absorbing ball in $H^{1}$ within approximately $N_{1} \approx N_{0}+\frac{2}{k}$ steps. This ends the proof of the uniform dissipativity of the approximations (6) by the compact imbedding of $H^{1}$ into $L^{2}$. This uniform 
bound also implies the existence of a compact global attractor $\mathcal{A}_{k} \subset H_{0, p e r}^{1}$ for the scheme for each time step $k \leq 8$ and

$$
\sup _{\theta \in K}\|\nabla \theta\| \leq \sqrt{c_{7}} .
$$

There are another three consequences of (35), which are: an $L^{2}\left(H^{2}\right)$ estimate on the solution, an estimate on the difference of the solution in the $H^{1}$ norm at adjacent steps, and a uniform-in-time $H^{1}$ norm estimate for initial data in $H^{1}$, i.e.,

$$
\begin{aligned}
\frac{1}{N} \sum_{n=0}^{N}\left\|\Delta \theta_{k}^{n+1}\right\|^{2} & \leq \frac{\left\|\nabla \theta_{0}\right\|^{2}}{k N}+2 c_{6}\left(1+R^{2}\right)^{6}, \\
\sum_{n=0}^{N}\left\|\nabla\left(\theta_{k}^{n+1}-\theta_{k}^{n}\right)\right\|^{2} & \leq\left\|\nabla \theta_{0}\right\|^{2}+2 c_{6}\left(1+R^{2}\right)^{6} k N, \\
\left\|\nabla \theta_{k}^{n+1}\right\|^{2} & \leq(1+k)^{-n+1}\left\|\nabla \theta_{0}\right\|^{2}+2 c_{6}\left(1+R^{2}\right)^{6} .
\end{aligned}
$$

These estimates will be useful in our uniform convergence proof.

Uniform estimates in Sobolev spaces of higher orders can be derived just as in the case of a continuous-in-time system. Here we sketch the proof that the $H^{2}$ norm of the solution is asymptotically uniformly bounded in time, i.e., that there is an absorbing ball in $H^{2}$ which attracts all solutions with $L^{2}$ initial data uniformly for all $k$.

For this purpose we apply $\Delta$ to both sides of the scheme (21) and then multiply the scheme by $\Delta \theta_{k}^{n+1}$ and integrate over the domain. This leads to the following:

$$
\begin{aligned}
\frac{1}{2 k}(\| & \left.\Delta \theta_{k}^{n+1}\left\|^{2}-\right\| \Delta \theta_{k}^{n}\left\|^{2}+\right\| \Delta\left(\theta_{k}^{n+1}-\theta_{k}^{n}\right) \|^{2}\right)+\left\|\nabla \Delta \theta_{k}^{n+1}\right\|^{2} \\
\leq & \left\|\tau^{(4)}\right\|\left\|\Delta \theta_{k}^{n+1}\right\|+R a\left(\left\|\Delta\left(A^{-1}\left(\mathbf{k} \theta_{k}^{n}\right)\right)\right\|_{L^{6}}\left\|\nabla \theta_{k}^{n+1}\right\|_{L^{3}}\right. \\
& \left.+2\left\|\nabla A^{-1}\left(\mathbf{k} \theta_{k}^{n}\right)\right\|_{L^{\infty}}\left\|\nabla^{2} \theta_{k}^{n+1}\right\|\right)\left\|\Delta \theta_{k}^{n+1}\right\|+R a\left(\left\|\Delta\left(A^{-1}\left(\mathbf{k} \theta_{k}^{n}\right)\right)\right\|\left\|\tau^{\prime}\right\|_{L^{\infty}}\right. \\
& \left.+2\left\|\nabla\left(A^{-1}\left(\mathbf{k} \theta_{k}^{n}\right)\right)\right\|\left\|\nabla \tau^{\prime}\right\|_{L^{\infty}}+\left\|A^{-1}\left(\mathbf{k} \theta_{k}^{n}\right)\right\|\left\|\Delta \tau^{\prime}\right\|_{L^{\infty}}\right)\left\|\Delta \theta_{k}^{n+1}\right\| \\
\leq & c_{8}\left(\left\|\Delta \theta_{k}^{n+1}\right\|+\left\|\Delta \theta_{k}^{n+1}\right\|^{2}\right) \\
\leq & c_{9}\left(\left\|\Delta \theta_{k}^{n+1}\right\|+\left\|\nabla \Delta \theta_{k}^{n+1}\right\|\left\|\nabla \theta_{k}^{n+1}\right\|\right) \\
\leq & \frac{1}{2}\left\|\nabla \Delta \theta_{k}^{n+1}\right\|^{2}+c_{10},
\end{aligned}
$$

where we have applied the identify $\int_{\Omega} A^{-1}\left(\mathbf{k} \theta_{k}^{n}\right) \nabla \Delta \theta_{k}^{n+1} \Delta \theta_{k}^{n+1}=0$, the fact that $\Delta \theta_{k}^{n+1}=0$ at $z=0,1$ (which follows from (21) 22)), Hölder's inequality, elliptic regularity, Sobolev imbedding, Cauchy-Schwarz, interpolation inequality, and the $H^{1}$ uniform estimate (38).

This leads to the uniform $H^{2}$ estimate.

To summarize, we have the following result.

Proposition 2 (Uniform dissipativity). There exists a constant $c_{10}$ independent of the time step $k$ such that the scheme (21) possesses an absorbing ball in $H^{1}$ and $H^{2}$ with radius $2 \sqrt{c_{10}}$ which attracts all bounded sets in $L^{2}$. In particular, we have

$$
\sup _{\mathbf{u} \in K}\|\mathbf{u}\|_{H^{2}} \leq 2 \sqrt{c_{10}},
$$

where $K$ is the union of the global attractors for the scheme with different time steps $k$. 
3.3. Finite time uniform convergence. We now verify the second condition (H2) in the main theorem. In fact, we will prove a slightly more general version which covers both $\mathrm{H} 2$ and H5, i.e., the convergence of the numerical scheme (21) on any finite time interval uniformly for initial data from $K=\bigcup \mathcal{A}_{k}$ (which is uniformly bounded in $H^{1}$ thanks to (38)).

For this purpose, we rewrite the scheme (21) as

$$
\begin{aligned}
\frac{\partial \tilde{\theta}_{k}(t)}{\partial t} & +R a A^{-1}\left(\mathbf{k} \theta_{k}(t)\right) \cdot \nabla \theta_{k}(t+k) \\
& +R a A^{-1}\left(\mathbf{k} \theta_{k}(t)\right)_{3} \tau^{\prime}(z)=\Delta \theta_{k}(t+k)+\tau^{\prime \prime}(z)
\end{aligned}
$$

where

$$
\begin{aligned}
& \theta_{k}(t)=\theta_{k}^{n}, t \in[n k,(n+1) k), \\
& \tilde{\theta}_{k}(t)=\theta_{k}^{n}+\frac{t-n k}{k}\left(\theta_{k}^{n+1}-\theta_{k}^{n}\right), t \in[n k,(n+1) k) .
\end{aligned}
$$

For any fixed $T^{*}>0$, the estimates (41, 39) imply that $\theta_{k}$ and $\tilde{\theta}_{k}$ are uniformly (in $k$ ) bounded in $L^{\infty}\left(0, T^{*} ; H^{1}\right)$ and $L^{2}\left(0, T^{*} ; H^{2}\right)$ and the bounds are uniform in $k$ and $\theta_{0} \in K$.

We can rephrase the differential form of the scheme as

$$
\begin{aligned}
\frac{\partial \tilde{\theta}_{k}(t)}{\partial t} & +R a A^{-1}\left(\mathbf{k} \tilde{\theta}_{k}(t)\right) \cdot \nabla \tilde{\theta}_{k}(t) \\
& +R a A^{-1}\left(\mathbf{k} \tilde{\theta}_{k}(t)\right)_{3} \tau^{\prime}(z)=\Delta \tilde{\theta}_{k}(t)+\tau^{\prime \prime}(z)+f_{k}(t),
\end{aligned}
$$

where

$$
\begin{aligned}
f_{k}(t)= & R a A^{-1}\left(\mathbf{k} \tilde{\theta}_{k}(t)\right) \cdot \nabla \tilde{\theta}_{k}(t)-R a A^{-1}\left(\mathbf{k} \theta_{k}(t)\right) \cdot \nabla \theta_{k}(t+k) \\
& +R a A^{-1}\left(\mathbf{k}\left(\tilde{\theta}_{k}(t)-\theta_{k}(t)\right)\right)_{3} \tau^{\prime}(z)-\Delta\left(\tilde{\theta}_{k}(t)-\theta_{k}(t+k)\right) .
\end{aligned}
$$

It is easy to see that $f_{k}$ is small due to our estimate on the difference of the approximate solution at adjacent time steps (40). Indeed, we have, for $t \in$ $[n k,(n+1) k)$,

$$
\begin{aligned}
\tilde{\theta}_{k}(t)-\theta_{k}(t+k) & =\frac{t-(n+1) k}{k}\left(\theta_{k}^{n+1}-\theta_{k}^{n}\right), \\
\tilde{\theta}_{k}(t)-\theta_{k}(t) & =\frac{t-n k}{k}\left(\theta_{k}^{n+1}-\theta_{k}^{n}\right)
\end{aligned}
$$

and therefore, for $\theta_{0} \in K$,

$$
\begin{aligned}
R a\left\|A^{-1}\left(\mathbf{k} \tilde{\theta}_{k}(t)\right) \cdot \nabla\left(\tilde{\theta}_{k}(t)-\theta_{k}(t+k)\right)\right\|_{H^{-1}} & \leq R a\left\|A^{-1}\left(\mathbf{k} \tilde{\theta}_{k}(t)\right)\right\|_{L^{\infty}}\left\|\theta_{k}^{n+1}-\theta_{k}^{n}\right\| \\
\left.R a \| A^{-1}\left(\mathbf{k}\left(\tilde{\theta}_{k}(t)-\theta_{k}(t)\right)\right) \cdot \nabla \theta_{k}(t+k)\right) \|_{H^{-1}} & \leq c_{11}\left\|\theta_{k}^{n+1}-\theta_{k}^{n}\right\|, \\
\leq R a\left\|A^{-1}\left(\mathbf{k}\left(\tilde{\theta}_{k}(t)-\theta_{k}(t)\right)\right)\right\|_{L^{\infty}}\left\|\theta_{k}(t+k)\right\| & \leq c_{12}\left\|\theta_{k}^{n+1}-\theta_{k}^{n}\right\|, \\
R a \| A^{-1}\left(\mathbf{k}\left(\tilde{\theta}_{k}(t)-\theta_{k}(t)\right)_{3} \tau^{\prime} \|_{H^{-1}}\right. & \leq c_{13}\left\|\theta_{k}^{n+1}-\theta_{k}^{n}\right\|, \\
\left\|\Delta\left(\tilde{\theta}_{k}(t)-\theta_{k}(t+k)\right)\right\|_{H^{-1}} & \leq c_{14}\left\|\nabla\left(\theta_{k}^{n+1}-\theta_{k}^{n}\right)\right\|,
\end{aligned}
$$

which further leads to

$$
\left\|f_{k}(t)\right\|_{H^{-1}} \leq c_{15}\left\|\nabla\left(\theta_{k}^{n+1}-\theta_{k}^{n}\right)\right\|, t \in[n k,(n+1) k)
$$


and hence, when combined with the estimate on the time difference estimate (40),

$$
\left\|f_{k}\right\|_{L^{2}\left(0, T^{*} ; H^{-1}\right)} \leq c_{15} \sqrt{\sum_{n=0}^{\frac{T^{*}}{k}} k\left\|\nabla\left(\theta_{k}^{n+1}-\theta_{k}^{n}\right)\right\|^{2}} \leq c_{16} \sqrt{k} .
$$

Taking the difference of the infinite Prandtl number model (19) and the differential form of the scheme (46), denoting $\xi_{k}(t)=\theta(t)-\tilde{\theta}_{k}(t)$, we have

$$
\begin{aligned}
\frac{\partial \xi_{k}(t)}{\partial t} & +R a A^{-1}(\mathbf{k} \theta(t)) \cdot \nabla \xi_{k}(t)+R a A^{-1}\left(\mathbf{k} \xi_{k}(t)\right) \cdot \nabla \tilde{\theta}_{k}(t) \\
& +R a A^{-1}\left(\mathbf{k} \xi_{k}(t)\right)_{3} \tau^{\prime}(z)=\Delta \xi_{k}(t)-f_{k}(t) .
\end{aligned}
$$

Multiplying this equation by $\xi_{k}$ and integrating over $\Omega$ we have

$$
\begin{aligned}
\frac{1}{2} \frac{d}{d t}\left\|\xi_{k}(t)\right\|^{2}+\left\|\nabla \xi_{k}(t)\right\|^{2} \\
\leq \\
\quad R a\left\|A^{-1}\left(\mathbf{k} \xi_{k}(t)\right)\right\|_{L^{\infty}}\left\|\nabla \tilde{\theta}_{k}(t)\right\|\left\|\xi_{k}(t)\right\|+R a\left\|A^{-1}\left(\mathbf{k} \xi_{k}(t)\right)_{3}\right\|_{L^{\infty}}\left\|\tau^{\prime}\right\|\left\|\xi_{k}(t)\right\| \\
\quad+\left\|f_{k}(t)\right\|_{H^{-1}}\left\|\nabla \xi_{k}(t)\right\| \\
\leq c_{17}\left\|\xi_{k}(t)\right\|^{2}+\frac{1}{2}\left\|f_{k}(t)\right\|_{H^{-1}}^{2}+\frac{1}{2}\left\|\nabla \xi_{k}(t)\right\|^{2} .
\end{aligned}
$$

Therefore, we have

$$
\frac{d}{d t}\left\|\xi_{k}(t)\right\|^{2} \leq 2 c_{17}\left\|\xi_{k}(t)\right\|^{2}+\left\|f_{k}(t)\right\|_{H^{-1}}^{2}, \quad\left\|\xi_{k}(0)\right\|=0
$$

which leads to

$$
\left\|\theta-\tilde{\theta}_{k}\right\|_{L^{\infty}\left(0, T^{*} ; L^{2}\right)}=\left\|\xi_{k}\right\|_{L^{\infty}\left(0, T^{*} ; L^{2}\right)} \leq c_{18}\left\|f_{k}\right\|_{L^{2}\left(0, T^{*} ; H^{-1}\right)} \leq c_{19} \sqrt{k} \rightarrow 0
$$

uniformly for $\theta_{0} \in K$.

This ends the proof of the finite time uniform convergence, which further implies H2 and H5. To summarize, we have the following result.

Proposition 3 (Finite time uniform convergence). For any $T^{*}>0$, there exists a constant $c_{19}$ independent of the time step $k$ such that

$$
\left\|\theta(n k)-\theta_{k}^{n}\right\| \leq c_{19} \sqrt{k}, \forall \theta_{0} \in K, \forall n k \leq T^{*},
$$

i.e., assumptions H2 (7) and H5 (14) are valid for the scheme (21) with $t_{0}=0$.

3.4. Finite time uniform continuity. Now we verify the finite time uniform continuity of the infinite Prandtl number model for initial data starting from $K$, the union of the global attractors of the scheme with different step size $k$.

It is easy to check that the $L^{2}$ norm of the infinite Prandtl number model (19) is uniformly bounded for $\theta_{0}$ belonging to a bounded set in $L^{2}$ (see for instance 8], and the discrete version (29)). A uniform $H^{1}$ norm estimate can also be derived (see (38) for the discrete version). Indeed, multiplying the infinite Prandtl number 
model (19) by $-\Delta \theta$ and integrating over $\Omega$, we deduce, for $\theta_{0} \in K$,

$$
\begin{aligned}
\frac{1}{2} \frac{d}{d t} \| & \nabla \theta(t)\left\|^{2}+\right\| \Delta \theta(t) \|^{2} \\
\leq & \left\|\tau^{\prime \prime}\right\|\|\Delta \theta(t)\|+R a\left\|A^{-1}(\mathbf{k} \theta(t))\right\|_{L^{\infty}}\|\nabla \theta(t)\|\|\Delta \theta(t)\| \\
& +R a\left\|A^{-1}(\mathbf{k} \theta(t))_{3}\right\|_{L^{\infty}}\left\|\tau^{\prime}\right\|\|\Delta \theta(t)\| \\
\leq & c_{20}(1+\|\theta(t)\|+\|\theta(t)\|\|\nabla \theta(t)\|)\|\Delta \theta(t)\| \\
\leq & \frac{1}{2}\|\Delta \theta(t)\|^{2}+c_{21}\left(1+\|\nabla \theta(t)\|^{2}\right) .
\end{aligned}
$$

This leads to the following estimates:

$$
\begin{aligned}
\|\theta\|_{L^{\infty}\left(0, T^{*} ; H^{1}\right)} & \leq c_{22}, \\
\|\theta\|_{L^{2}\left(0, T^{*} ; H^{2}\right)} & \leq c_{22} .
\end{aligned}
$$

Now, integrating the infinite Prandtl number model (19) in time from $t$ to $T^{*}$ we have, for $\theta_{0} \in K$,

$$
\begin{aligned}
\| S(t) & \theta_{0}-S\left(T^{*}\right) \theta_{0} \| \\
\leq & \mid \int_{t}^{T^{*}}\left(\|\Delta \theta(s)\|+R a\left\|A^{-1}(\mathbf{k} \theta(s))\right\|_{L^{\infty}}\|\nabla \theta(s)\|\right. \\
& \left.+R a\left\|A^{-1}(\mathbf{k} \theta(s))_{3}\right\|_{L^{\infty}}\left\|\tau^{\prime}\right\|+\left\|\tau^{\prime \prime}\right\|\right) d s \mid \\
\leq & \left|\int_{t}^{T^{*}}\left(\|\Delta \theta(s)\|+c_{23}(1+\|\theta(s)\|\|\nabla \theta(s)\|+\|\theta(s)\|)\right) d s\right| \\
\leq & \left|\int_{t}^{T^{*}}\left(\|\Delta \theta(s)\|+c_{24}\right) d s\right| \\
\leq & c_{25} \sqrt{\left|T^{*}-t\right|},
\end{aligned}
$$

where we have used elliptic regularity, the uniform $H^{1}$ estimate, and the $L^{2}\left(H^{2}\right)$ estimate.

This completes the proof of the uniform continuity of the infinite Prandtl number model, i.e., H3. To summarize, we have the following result.

Proposition 4 (Finite time uniform continuity). For any $T^{*}>0$, the infinite Prandtl number model (19) is continuous on the time interval $\left[0, T^{*}\right]$ uniformly for initial data from the union of the global attractors $K$ defined in (6).

\section{COnClusions And REMARKS}

We have presented a general/abstract result on the convergence of stationary statistical properties of time approximation of infinite-dimensional dissipative dynamical systems. The three natural conditions that guarantee the convergence of the stationary statistical properties are uniform dissipativity of the scheme, uniform convergence of the scheme on $[0,1]$, and the uniform continuity of the underlying continuous dynamical system on the unit time interval $[0,1]$. We hope that this work will stimulate further work on numerical schemes that are able to capture stationary statistical properties of infinite-dimensional dissipative systems.

We have also illustrated the application of our main result to the infinite Prandtl number model for convection and we believe that the abstract main result presented here is applicable to many other dissipative systems and associated schemes. Fully 
discretized approximation can be studied similarly for Galerkin-type spatial approximations that enjoy the three properties postulated (see [27, 28, 11] for fully discrete long time stable schemes for other equations). Numerical implementation in physically relevant regimes is non-trivial. Although the numerical scheme that we proposed here is linear, which is advantageous over non-linear schemes such as the one induced by the fully implicit Euler scheme proposed by many authors, the linear equation (the matrix in the fully discretized case) changes at each time step due to the presence of the convection term $R a A^{-1}\left(\mathbf{k} \theta_{k}^{n}\right) \cdot \nabla \theta_{k}^{n+1}$. This together with the need for long time simulation combined with the presence of physically expected small spatial scales of the order of $R a^{-\frac{1}{3}}$ or $R a^{-\frac{1}{2}}$ [3, 7, 19] which need to be resolved makes it a challenge to simulate the physically interesting large Rayleigh number regime. One of the immediate goals is to design more efficient numerical schemes than the one presented here. We will report this and our numerical results elsewhere.

\section{ACKNOWLEDGEMENT}

The author acknowledges helpful conversations with Andrew Majda, Jie Shen and Joe Tribbia. This work is supported in part by the National Science Foundation through DMS0606671 and a 111 project from the Chinese Ministry of Education. He also thanks an anonymous referee for his/her constructive comments which helped to improve the presentation.

\section{REFERENCES}

1. Billingsley, P., Weak convergence of measures: Applications in probability. SIAM, Philadelphia, 1971. MR0310933 (46:10031)

2. Cances, Eric; Legoll, Frederic; Stoltz, Gabriel, Theoretical and numerical comparison of some sampling methods for molecular dynamics. ESAIM: Mathematical Modelling and Numerical Analysis, 41, no. 2 (2007), pp. 351-389. MR2339633 (2008e:82038)

3. Chandrasekhar, S., Hydrodynamic and hydromagnetic stability. Oxford, Clarendon Press, 1961. MR0128226 (23:B1270)

4. Cheng, W.; Wang, X., A uniformly dissipative scheme for stationary statistical properties of the infinite Prandtl number model, Applied Mathematics Letters, 21 (2008), no. 12, 1281-1285. MR 2464380

5. Cheng, W.; Wang, X., A semi-implicit scheme for stationary statistical properties of the infinite Prandtl number model, SIAM J. Numer. Anal., vol. 47, no.1, 250-270, 2008. DOI.10.1137/080713501. MR 2452860

6. Chiu, C.; Du, Q.; Li, T.Y., Error estimates of the Markov finite approximation of the Frobenius-Perron operator, Nonlinear Anal. 19(1992), no.4, 291-308. MR 1178404 (93f:28014)

7. Constantin, P.; Doering, C.R., Infinite Prandtl number convection, J. Stat. Phys. 94 (1999), no. 1-2, 159-172. MR1679670 (2000a:76152)

8. Doering, C.R.; Otto, F.; Reznikoff, M.G., Bounds on vertical heat transport for infinite Prandtl number Rayleigh-Bénard convection, J. Fluid Mech. (2006), vol. 560, pp. 229-241. MR 2265709 (2008b:76171)

9. E, W.; Li, D., The Andersen thermostat in molecular dynamics, Comm. Pure Appl. Math. 61 (2008), 96-136. MR 2361305 (2008j:82047)

10. Foias, C., Jolly, M., Kevrekidis, I.G. and Titi, E.S., Dissipativity of numerical schemes, Nonlinearity 4 (1991), 591-613. MR1124326 (92f:65099)

11. Foias, C., Jolly, M., Kevrekidis, I.G. and Titi, E.S., On some dissipative fully discrete nonlinear Galerkin schemes for the Kuramoto-Sivashinsky equation, Phys. Lett. A 186 (1994), 87-96. MR.1267024(94k:35259)

12. Foias, C.; Manley, O.; Rosa, R.; Temam, R., Navier-Stokes equations and turbulence. Encyclopedia of Mathematics and its Applications, 83. Cambridge University Press, Cambridge, 2001. MR.1855030 (2003a:76001) 
13. Geveci, T., On the convergence of a time discretization scheme for the Navier-Stokes equations, Math. Comp., 53 (1989), pp. 43-53. MR969488 (89m:65085)

14. Hale, J. K., Asymptotic behavior of dissipative systems, American Mathematical Society, Providence, RI, 1988. MR941371 (89g:58059)

15. Heywood, J. G.; Rannacher, R.; Finite element approximation of the nonstationary NavierStokes problem. II. Stability of solutions and error estimates uniform in time. SIAM J. Numer. Anal. 23 (1986), no. 4, 750-777. MR 849281 (88b:65132)

16. Hill, A.T.; Süli, E., Approximation of the global attractor for the incompressible Navier-Stokes equations, IMA J. Numer. Anal., 20 (2000), 663-667. MR1795301(2001j:37138)

17. Jones, D.A.; Stuart, A.M.; Titi, E.S., Persistence of Invariant Sets for Dissipative Evolution Equations, J. Math. Anal. Appl., 219, 479-502 (1998). MR1606370 (98m:34123)

18. Ju, N., On the global stability of a temporal discretization scheme for the Navier-Stokes equations, IMA J. Numer. Anal., 22 (2002), pp. 577-597. MR1936521(2003g:76087)

19. Kadanoff, L.P., Turbulent heat flow: Structures and scaling, Physics Today, 54, no. 8, pp. 34-39, 2001.

20. Larsson, S., The long-time behavior of finite-element approximations of solutions to semilinear parabolic problems. SIAM J. Numer. Anal. 26 (1989), no. 2, 348-365. MR987394 (90g:65124)

21. Lasota, A.; Mackey, M.C., Chaos, Fractals, and Noise, Stochastic Aspects of Dynamics, 2nd, ed., Springer-Verlag, New York, 1994. MR1244104 (94j:58102)

22. Lax, P.D., Functional Analysis, Wiley, New York, 2002. MR.1892228 (2003a:47001)

23. Majda, A.J.; Wang, X., Nonlinear Dynamics and Statistical Theory for Basic Geophysical Flows, Cambridge University Press, Cambridge, England, (2006). MR 2241372

24. Monin, A.S.; Yaglom, A.M., Statistical fluid mechanics; mechanics of turbulence, English ed. updated, augmented and rev. by the authors. MIT Press, Cambridge, Mass., 1975.

25. Raugel, G., Global attractors in partial differential equations. Handbook of dynamical systems, Vol. 2, 885-982, North-Holland, Amsterdam, 2002. MR1901068 (2003f:37151)

26. Reich, S., Backward error analysis for numerical integrators. SIAM J. Numer. Anal., vol. 36, no. 5, pp. 1549-1570, 1999. MR.1706731 (2000f:65060)

27. Shen, J., Convergence of approximate attractors for a fully discrete system for reactiondiffusion equations, Numer. Funct. Anal. and Optimiz., 10 (11 \& 12) pp. 1213-1234 (1989). MR 1050711 (92d:65155)

28. Shen, J., Long time stability and convergences for the fully discrete nonlinear Galerkin methods, Appl. Anal., 38 (1990), pp. 201-229. MR.1116181(93a:65130)

29. Sigurgeirsson, H.; Stuart, A.M., Statistics from computations, in Foundations of Computational Mathematics, Edited by R. Devore, A. Iserles and E. Suli, pp. 323-344, Cambridge University Press, 2001. MR1839148

30. Stuart, A.M.; Humphries, A.R., Dynamical Systems and Numerical Analysis. Cambridge University Press, 1996. MR1402909 (97g:65009)

31. Temam, R.M., Navier-Stokes Equations and Nonlinear Functional Analysis, 2nd edition, CBMS-SIAM, SIAM, 1995. MR1318914 (96e:35136)

32. Temam, R.M., Infinite Dimensional Dynamical Systems in Mechanics and Physics, 2nd ed. Springer-Verlag, New York, 1997. MR1441312 (98b:58056)

33. Tone, F.; Wirosoetisno, D., On the long-time stability of the implicit Euler scheme for the two-dimensional Navier-Stokes equations. SIAM J. Num. Anal. 44 (2006), no. 1, 29-40 MR2217369(2006m:65184)

34. Tritton, D.J., Physical Fluid Dynamics. Oxford Science Publishing, 1988. MR1047471 (93f:76001)

35. Tupper, P.F., Ergodicity and the numerical simulation of Hamiltonian systems. SIAM J. Applied Dynamical Systems, vol. 4, no.3, pp. 563-587, 2005. MR2145198(2006i:65227)

36. Vishik, M.I.; Fursikov, A.V., Mathematical Problems of Statistical Hydromechanics. Kluwer Acad. Publishers, Dordrecht/Boston/London, 1988.

37. Walters, P., An introduction to ergodic theory. Springer-Verlag, New York, 2000. MR648108 $(84 \mathrm{e}: 28017)$

38. Wang, X., Infinite Prandtl Number Limit of Rayleigh-Bénard Convection. Comm. Pure and Appl. Math., vol. 57, issue 10, (2004), 1265-1282. MR2069723(2005d:76015)

39. Wang, X., Stationary statistical properties of Rayleigh-Bénard convection at large Prandtl number. Comm. Pure and Appl. Math., 61 (2008), no. 6, 789-815. DOI: 10.1002/cpa.20214. MR2400606 
40. Wang, X., Upper Semi-Continuity of Stationary Statistical Properties of Dissipative Systems. Dedicated to Prof. Li Ta-Tsien on the occasion of his 70th birthday. Discrete and Continuous Dynamical Systems, A. Vol. 23, no.1/2, pp. 521-540, 2009. doi:10.3934/dcds.2009.23.521 MR2449091

Department of Mathematics, Florida State University, Tallahassee, Florida 32306 and School of Mathematics, Fudan University, Shanghai, China 200433

E-mail address: wxm@math.fsu.edu 